\title{
Ectopia Cordis
}

\section{Reporte de un caso}

García-Muñoz Iván *, Flores-Álvarez Efrén ${ }^{* *}$, González-Peña Javier ***, Chagolla-Santillán Miguel Ángel ${ }^{* * *}$, Acevedo-Bañuelos lliana ${ }^{* * *}$; Rocha-Peña Elena ${ }^{* * * *}$

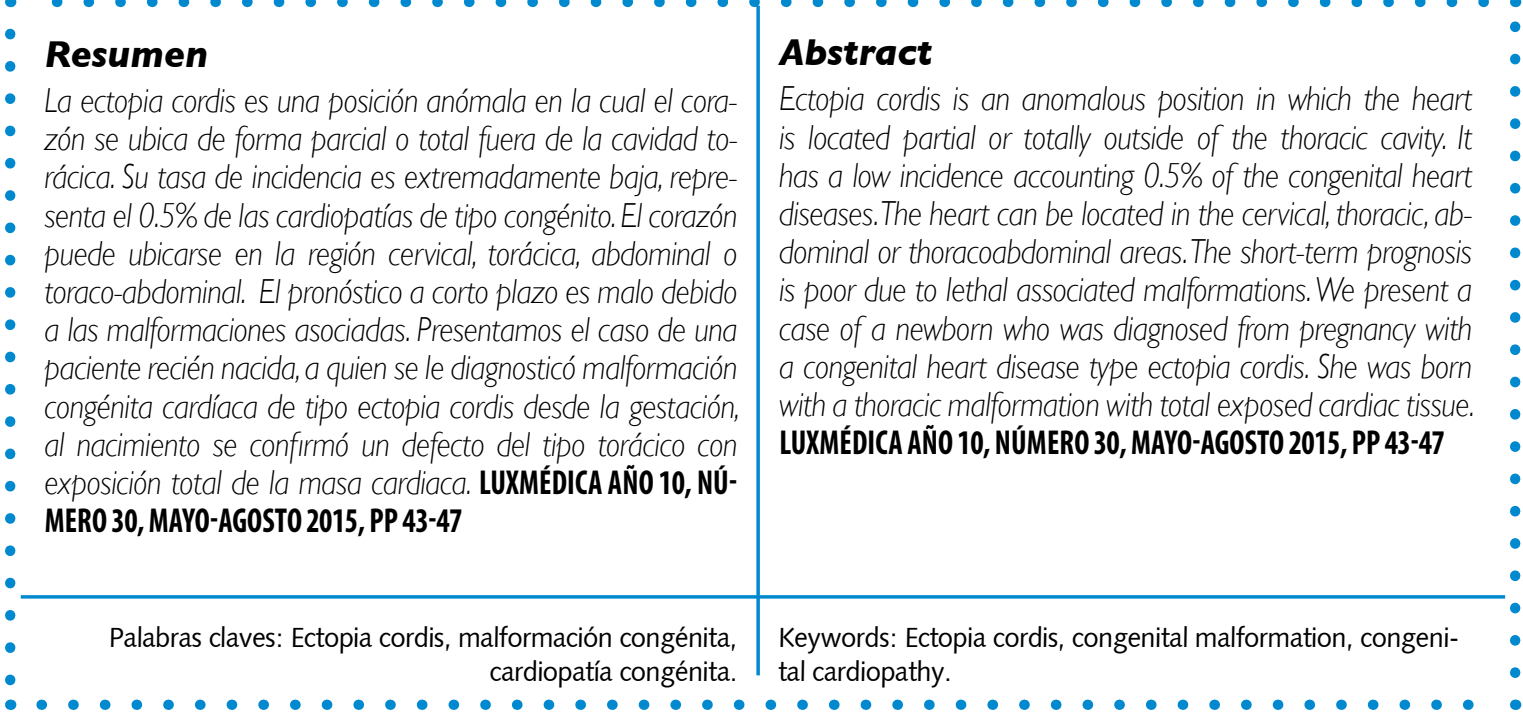

\section{Introducción}

La ectopia cordis se describió por primera vez en 1671, pero el término fue acuñado por Abbott ${ }^{1}$ en 1898. Es una anomalía congénita cardíaca que consiste en una posición cardíaca fuera de la caja torácica en forma parcial o total. Es una malformación extremadamente rara, se ha reportado una incidencia que fluctúa entre 5.5 a 7.9 casos por millón de nacidos vivos y representa el $0.5 \%$

\footnotetext{
* Residente de Cirugía Cardiovascular. Antiguo Hospital Civil de Guadalajara Fray Antonio Alcalde.

** Profesor Titular de Posgrado en Cirugía General. Centenario Hospital Miguel Hidalgo.

*** Adscrito al servicio de Cirugía Cardiovascular. Hospital Regional de Alta Especialidad Bajío.

***** Médico General.

Fecha de recibido: 2 de junio 2015

Fecha de aceptación: 7 de julio 2015

Correspondencia: MCM Efrén Flores Álvarez, Departamento de Cirugía, Centenario Hospital Miguel Hidalgo, Calle Galeana Sur Número 465, Colonia Obraje, Código Postal 20230, Aguascalientes, Aguascalientes, México. Teléfono 014499946720 correo electrónico: efflorez@hotmail.com
} 


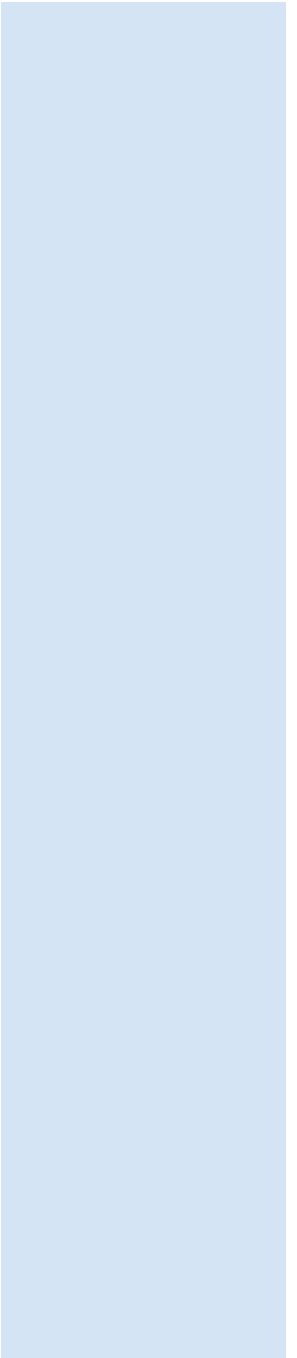

de las cardiopatías congénitas. Ocurre con mayor frecuencia en el sexo femenino. ${ }^{2}$

De acuerdo a la localización del tejido cardíaco al nacimiento se reconocen 4 variedades: cervical, torácica, tóraco-abdominal y abdominal. Se considera que su origen es debido a una falla en la maduración de los componentes mesodérmicos de la línea media del tórax y del abdomen. El pronóstico es malo a corto plazo pues influye en el tipo de ectopia, las malformaciones asociadas y las complicaciones correspondientes, que suelen ser letales por naturaleza. $^{3}$

La variedad cervical ha sido descrita solamente en fetos malformados. Sólo existe un caso reportado en la literatura mundial de la variedad abdominal. La variedad toraco-abdominal es una forma parcial de ectopia cordis caracterizada por una ausencia parcial o una hendidura de la porción inferior del esternón, ausencia del pericardio diafragmático parietal y un defecto de la línea media del diafragma, originando una comunicación libre entre el pericardio y la cavidad abdominal, con diástasis de los músculos rectos y desplazamiento parcial de la porción ventricular dentro del epigastrio. Existen reportes con sobrevida en la mitad de los pacientes para esta variedad. ${ }^{4,5}$

La variedad torácica es la forma clásica de ectopia cordis, en estos pacientes hay una hendidura en el esternón que permite la protrusión del corazón hacia fuera de la cavidad torácica, con pérdida del pericardio parietal, orientación cefálica del ápex cardiaco, onfalocele epigástrico, diástasis de los músculos rectos y una cavidad torácica pequeña. Generalmente se asocia a defectos cardiacos complejos con alteraciones en la conexión atrio-ventricular y ventrículo-arterial; los pacientes no suelen sobrevivir más de 36 horas. ${ }^{5-7}$

\section{Presentación del caso clínico}

Presentamos el caso de un recién nacido, de sexo femenino, pre-término, con adecuado control prenatal desde el primer trimestre de gestación. A la semana 20 de la gestación se le detectó la presencia de malformaciones congénitas. Por eco-cardiografía se le encontró un defecto cardíaco severo compatible con ectopia cordis asociado a tetralogía de Fallot.

A pesar de las malformaciones cursó con una gestación normo-evolutiva, sin ningún tipo de complicación. A la semana 36 , fue obtenida mediante una operación cesárea. Al nacimiento destacaban los siguientes datos clínicos: peso de 2350 gr., puntaje de Apgar de 5 y 7, cianosis de piel y mucosas, polipnea con tiraje subcostal y se confirmó la presencia de una cardiopatía congénita con un defecto del tipo torácico con exposición total de la masa cardiaca (fig. 1). 
Se protegió la masa cardíaca con material sintético y se realizó aislamiento del medio ambiente mediante la colocación de un silo. (fig. 2 y 3 )

El paciente fue trasladado a la unidad de terapia neonatal, su evolución no fue favorable con deterioro general progresivo, con polipnea y taquicardia. No obstante el manejo intensivo, falleció a las 24 horas de su nacimiento.

\section{Discusión}

La ectopia cordis se origina por defectos segmentarios del desarrollo mesodérmico durante la tercera semana de la vida intrauterina y por alteraciones de las bandas amnióticas, que producen simultáneamente malformaciones cerebrales y toracoabdominales. Rara vez la ectopia cordis se asocia a alteraciones cromosómicas. ${ }^{8}$

La existencia de ectopia cordis con cardiopatía severa se ha confirmado en edad perinatal mediante ecocardiografía vaginal a las 10-12 semanas de gestación o por vía abdominal a las $20-22$ semanas. ${ }^{9}$ El tipo de cardiopatía que ocurre con más frecuencia es la comunicación interventricular, presente en el $59 \%$ de los casos, seguido de la comunicación interauricular en el $35 \%$, estenosis o atresia pulmonar en el $36 \%$, tetralogía de Fallot en el $22 \%$, divertículo del ventrículo derecho en el $13 \%$, vena cava superior izquierda en el $12 \%$ y doble salida del ventrículo derecho en el $13 \%$. Otras cardiopatías, como el ventrículo único, la transposición de grandes vasos y el defecto atrio ventricular completo se presentan rara vez. ${ }^{3,6,10}$

La ectopia cordis se puede acompañar o formar parte de un considerable número de malformaciones tanto cardiacas como extra cardiacas que involucran la línea media y que van desde el cráneo con exencefalia, labio y paladar hendido. En el tórax coexisten defectos del esternón anterior, del pericardio, del corazón y malformaciones pulmonares. En el abdomen común- mente se presentan defectos anatómicos como el onfalocele, la hernia diafragmática y otras malformaciones gastrointestinales y renales. Los defectos extra cardiacos que aumentan el riesgo son la hipoplasia pulmonar, las anomalías cerebrales y la herniación del hígado y otras vísceras al tó$\operatorname{rax}^{5,11}$

El manejo perinatal de la ectopia cordis estará orientado al diagnóstico preferentemente prenatal detallado de este defecto como de otros acompañantes, a fin de definir tanto el pronóstico como eventuales tratamientos. La ectopia cordis puede ser completa, por ausencia de piel y pericardio parietal, o parcial si existe debajo del esternón pericardio o encima del esternón piel. La existencia de un defecto parcial inferior o superior del esternón sin abertura total con presencia de pericardio parietal y piel favorece el tratamiento quirúrgico y evita la compresión torácica al introducir la víscera en la cavidad. En los últimos años se ha intentado la corrección quirúrgica en una o dos fases con resultados variables, dependientes fundamentalmente del tipo de cardiopatía asociada. ${ }^{12}$

La ectopia cordis se considera en términos generales como un síndrome con mal pronóstico a corto plazo, el cual mejora si hay pocos defectos asociados y el abordaje quirúrgico de esos defectos se hace tempranamente. El equipo médico multidisciplinario que involucra al neonatólogo, al pediatra, al cardiólogo pediatra, al cirujano pediatra plástico y al cirujano cardiovascu- 
lar, entre otros. Las técnicas quirúrgicas y el apoyo de la terapia intensiva neonatal han permitido que los pacientes sobrevivan al manejo quirúrgico que involucra tórax, corazón y abdomen. Al ser un síndrome poco frecuente, representa un reto para el grupo médico que debe cubrir el corazón expuesto, reducir hernias y cerrar los elementos de la línea media. En ocasiones en este primer tiempo se deben realizar cirugías cardiacas de carácter paliativo ya que, habitualmente, los defectos intracardíacos son múltiples y complejos y requieren varios tiempos quirúrgicos para su corrección o paliación definitiva. ${ }^{10,13,14}$

Hornberger y cols. ${ }^{15}$ reportaron una evolución favorable, tanto posquirúrgica inmediata como a largo plazo en cinco de diez pacientes con ectopia cordis y malformaciones intracardiacas. Ninguno de ellos tenía significativos defectos extra torácicos. En tres de los cinco fallecidos, la muerte se debió a un gran onfalocele e hipoplasia pulmonar, en uno a sepsis y uno

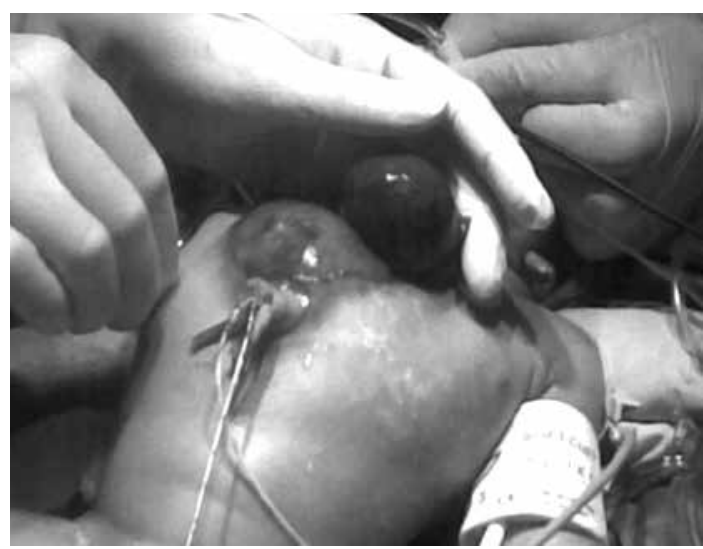

Fig. 1. Presencia de defecto del tipo torácico con exposición total de la masa cardiaca. falleció durante la cirugía cardíaca. Los autores concluyeron que pacientes con ectopia cordis, en ausencia de malformaciones extra torácicas y aún con un significativo defecto intracardiaco, pueden sobrevivir a la cirugía y presentar una buena evolución a largo plazo.

En general, podemos considerar a la ectopia cordis como una enfermedad congénita que consiste en una exposición del corazón a través de un defecto anterior del tronco, de extensión variable. El manejo ideal debe iniciar en la fase prenatal, debiendo incluir la delimitación del defecto de la pared torácica y la identificación de otras malformaciones para orientar a eventuales tratamientos y pronóstico. A pesar de la gravedad del cuadro, existen posibilidades de corrección quirúrgica y de sobrevida, dependiendo de la extensión del defecto y de las malformaciones asociadas. La presencia de un defecto intracardiaco no sería determinante para el pronóstico.

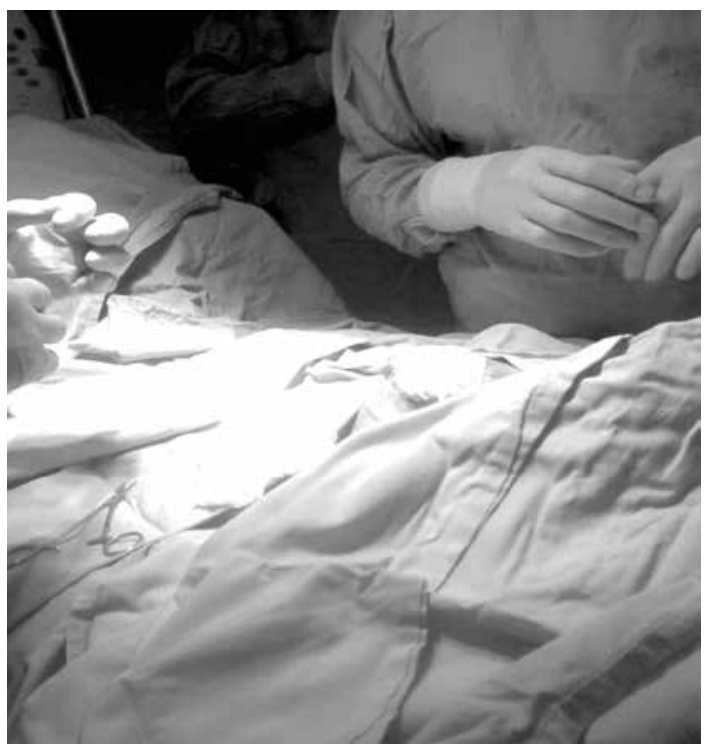

Fig. 2. Colocación de Parche de Goretex para cubrir el defecto en la pared torácica. 


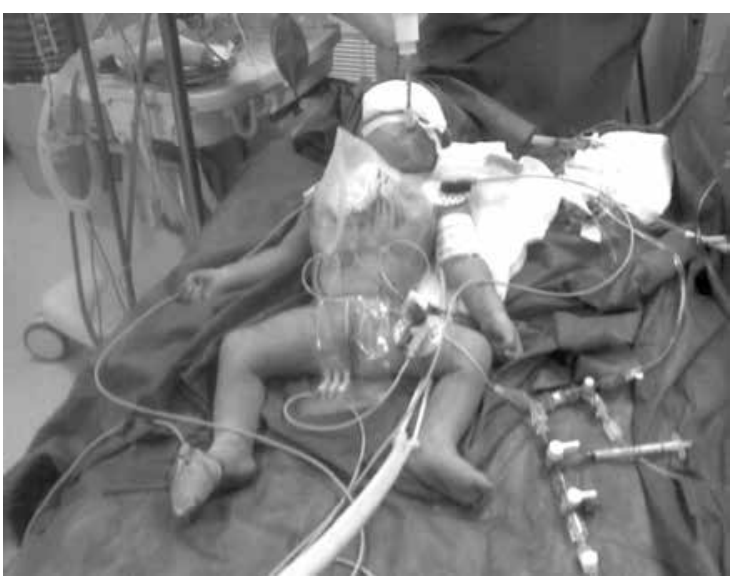

Fig. 3. Colocación de Silo y aislamiento al medio ambiente.

\section{Bibliografía}

1. Abbott FC. Congenital abnormality of sternum and diaphragm: protrusion of the heart in the epigastric region. Trans Pathol Soc London 1898;68:57-9.

2. Khoury MJ, Cordero JF, Rasmussen S. Ectopia cordis, midline defects and chromosome abnormalities: An epidemiologic perspective. Am J Med Genet 1988;30:811-17.

3. Cantrell JR, Haller JA, Ravitch MM. A syndrome of congenital defects involving the abdominal wall, sternum, diaphragm, pericardium, and heart. Surg Gynecol Obstet 1958;107:602-14.

4. Gabriel A, Donnelly J, Kuc A, Good D, Doros G, Matusz $P$, Loukas $M$. Ectopia cordis: a rare congenital anomaly. Clin Anat. 2014;27(8):1193-9.

5. Morales M, Patel S, Duff J, Simpson J, Villareal R. Ectopia cordis and other midline defects. Ann Thorac Surg 2000;70:111-4.

6. Cabrera A, Rodrigo D, Luis T, Pastor E, Galdeano JM, Esteban S. Anomalías cardiacas en la ectopia cordis. Rev Esp Cardiol 2002;55:1209-12.

7. Lubala TK, Mutombo AM, Katamea T, Lubala N, Munkana AN, Kabuya MS, Monga JK, Luboya ON. Sporadic thoracic ectopia cordis: clinical description of a case. Pan Afr Med J. 2012;13:62.

8. Sadler TW. The embryologic origin of ventral body wall defects. Semin Pediatr Surg. 2010;19(3):209-14.
9. Achiron $R$, Schimmel M, Farber B, Glaser J. Prenatal sonographic diagnosis and perinatal management of ectopia cordis. Ultrasound Obstet Gynecol 1991;1:431-4.

10. van Hoorn JHL, Moonen RMJ, Huysentruyt CJR, van Heurn LWE, Offermans JPM, Mulder ALMT. Pentalogy of Cantrell: two patients and a review to determine prognostic factors for optimal approach. Eur J Pediatr 2008;167:29-35.

11. Pepper MA, Fishbein GA, Teitell MA. Thoracoabdominal wall defect with complete ectopia cordis and gastroschisis: a case report and review of the literature. Pediatr Dev Pathol. 2013;16(5):348-52.

12. Lopez-Gutierrez JC. PHACES syndrome and ectopia cordis. Interact Cardiovasc Thorac Surg. 2011 Apr;12(4):642-4.

13. Gámez JM, de la Fuente A, García F. Ectopia cordis. An Pediatr . 2010;72(3):223.

14. Mohan $R$, Peralta $M$, Perez $R$, Rosenkranz ER, Panthaki ZJ. Chest wall reconstruction in a pediatric patient with ectopia cordis. Ann Plast Surg. 2010;65(2):211-3.

15. Hornberger LK, Colan SD, Lock JE. Outcome of patiens with ectopia cordis and significat intracardiac defects. Circulation 1996;94(suppl):32-7. 